\title{
Virtual screening and network pharmacology-based synergistic mechanism identification of multiple components contained in Guanxin V against coronary artery disease
}

\author{
Bo Liang ${ }^{1}$ (i), Xiao-Xiao Zhang ${ }^{1}$ and Ning Gu${ }^{2^{*}}$ (i)
}

\begin{abstract}
Background: Guanxin V (GXV), a traditional Chinese medicine (TCM), has been widely used to treat coronary artery disease (CAD) in clinical practice in China. However, research on the active components and underlying mechanisms of GXV in CAD is still scarce.

Methods: A virtual screening and network pharmacological approach was utilized for predicting the pharmacological mechanisms of GXV in CAD. The active compounds of GXV based on various TCM-related databases were selected and then the potential targets of these compounds were identified. Then, after the CAD targets were built through nine databases, a PPI network was constructed based on the matching GXV and CAD potential targets, and the hub targets were screened by MCODE. Moreover, Metascape was applied to GO and KEGG functional enrichment. Finally, HPLC fingerprints of GXV were established.

Results: A total of 119 active components and 121 potential targets shared between CAD and GXV were obtained. The results of functional enrichment indicated that several GO biological processes and KEGG pathways of GXV mostly participated in the therapeutic mechanisms. Furthermore, 7 hub MCODEs of GXV were collected as potential targets, implying the complex effects of GXV-mediated protection against CAD. Six specific chemicals were identified.

Conclusion: GXV could be employed for CAD through molecular mechanisms, involving complex interactions between multiple compounds and targets, as predicted by virtual screening and network pharmacology. Our study provides a new TCM for the treatment of CAD and deepens the understanding of the molecular mechanisms of GXV against CAD.
\end{abstract}

Keywords: Guanxin V, Coronary artery disease, Virtual screening, Network pharmacology, HPLC

*Correspondence: guning@njucm.edu.cn; jsguning@163.com

${ }^{2}$ Nanjing Hospital of Chinese Medicine Affiliated to Nanjing University of Chinese Medicine, Nanjing, China

Full list of author information is available at the end of the article

(c) The Author(s). 2020 Open Access This article is licensed under a Creative Commons Attribution 4.0 International License, which permits use, sharing, adaptation, distribution and reproduction in any medium or format, as long as you give appropriate credit to the original author(s) and the source, provide a link to the Creative Commons licence, and indicate if changes were made. The images or other third party material in this article are included in the article's Creative Commons licence, unless indicated otherwise in a credit line to the material. If material is not included in the article's Creative Commons licence and your intended use is not permitted by statutory regulation or exceeds the permitted use, you will need to obtain permission directly from the copyright holder. To view a copy of this licence, visit http://creativecommons.org/licenses/by/4.0/. The Creative Commons Public Domain Dedication waiver (http://creativecommons.org/publicdomain/zero/1.0/) applies to the data made available in this article, unless otherwise stated in a credit line to the data. 


\section{Background}

Even though interrelated changes in demography, environment, lifestyle, and health care, including the rising burden from coronary artery disease (CAD), indicate a transition in cardiovascular diseases epidemiology, cardiovascular diseases are still the leading cause of death worldwide [1, 2], posing a huge threat to global public health $[3,4]$. Among cardiovascular death, CAD accounts for a large part $[4,5]$. The treatment of CAD is mainly based on lifestyle management, drug treatment, and revascularization [6]. Guanxin V (GXV), an in-hospital preparation traditional Chinese medicine (TCM), has a significant effect on CAD [7-13], and our previous studies showed that GXV reduced serum inflammatory factor release $[10,14-17]$ through the TLR4/MyD88/NF-KB signaling pathway $[16,18,19]$. Network pharmacology, as a multidisciplinary science, reveals the pathophysiology and therapy strategies of numerous disorders by integrating related sciences [20-22], such as systems biology and pharmacology. Network pharmacology is of great significance for the discovery of effective components and potential targets in TCM and the investigation of its underlying mechanisms, which may help explore the pharmacological properties of herbal medicines [23, 24]. In view of the complex composition of TCM, it has the characteristics of multitarget, multichannel and coordination and synergism [25-28]. With the continuous improvement in the natural science system, the study of in-depth drug disease mechanisms is increasing [29]. However, due to the relatively backward research on the modernization of TCM theory, the integrity of TCM, the complexity of TCM components and the multichannel and multitarget mechanisms of the property, the material basis of the efficacy of TCM is not clear, and the mechanisms are not clear. At present, the mechanisms of GXV in CAD have not been fully elucidated, except for the previously mentioned mechanisms. In the present study, virtual screening and network pharmacology-based synergistic mechanism investigations of multiple components contained in GXV against CAD were systematically conducted.

\section{Materials and methods}

\section{Compounds in GXV}

The chemical compounds contained in the six herbs (Codonopsis Radix, Ophiopogon japonicus, Schizandra Chinensis Fructus, Rehmannia Radix Praeparata, Radix Salviae, and Radix Paeoniae Rubra) that constitute GXV were retrieved from TCMSP [30], TCMID [31], and BATMAN-TCM [32]. In addition, we combined the published literature to supplement the chemical compounds of the drugs contained in GXV.

\section{Active compounds of GXV}

The ADME characteristics of each obtained chemical compound were explored to obtain the bioactive compounds in six herbs of GXV. In our study, oral bioavailability (OB) [30, 33] and drug likeness (DL) [30,34], two commonly used ADME-related parameters, were assessed for each bioactive chemical compound. Generally, compounds with $\mathrm{OB} \geq 30 \%$ and $\mathrm{DL} \geq 0.18$ are considered to have pharmacological activities [35, 36].

\section{Targets of active compounds in GXV}

After obtaining the active compounds in GXV, the potential targets were also investigated from TCMSP [30], TCMID [31], and BATMAN-TCM [32]. Similarly, we combined the published literature to supplement the targets of active compounds in GXV.

\section{Targets in CAD}

The CAD-associated human targets were surveyed using diverse databases, including GeneCards [37], TTD [38], DrugBank [39], DisGeNET [40, 41], OMIM, TCMSP [30], MalaCards [42], NCBI, and CTD [43] with the search species limited to 'Homo sapiens'. Among them, candidate targets with correlation scores $\geq$ the mean in GeneCards [37] and gene disease correlation scores $\geq$ the mean in DisGeNET $[40,41]$ were included in the follow-up analysis. Furthermore, the potential targets were supplemented with relevant literature, and all the results were summarized and deduplicated.

\section{$\mathrm{H}-\mathrm{C}, \mathrm{H}-\mathrm{C}-\mathrm{T}$, and H-C-T-D networks}

The herb-compound ( $\mathrm{H}-\mathrm{C})$, herb-compound-target $(\mathrm{H}-\mathrm{C}$ $\mathrm{T}$ ), and herb-compound-target-disease (H-C-T-D) networks were established by connecting the corresponding elements [44]. The potential targets of the active compounds contained in GXV and the potential targets of CAD were annotated in Universal Protein (UniProt, https://www.uniprot.org/) [45]. The potential targets shared by the active compounds contained in GXV and CAD were applied for subsequent analysis. All networks were visualized in Cytoscape (version 3.7.1) [46].

\section{Functional enrichment}

Metascape [47] was applied to conduct enrichment analysis (including Gene Ontology (GO) terms [48] and Kyoto Encyclopedia of Genes and Genomes (KEGG) pathways [49]) of targets significantly associated with GXV and CAD, which was different from the previous study [50]. The list of annotations retrieved from the latest version of the Metascape database (last updated on 2019-08-14) was summarized in Table S1. When a term had $\geq 3$ counts, $>1.5$ enrichment factors, and $P<0.01$, it was seen as significant $[47,51]$. Moreover, for each given target, protein-protein interaction (PPI) enrichment 
analysis was carried out with three databases: BioGRID [52], InWeb_InBioMap [53], and OmniPath [54]. MCODE [55] was used to discover closely connected network components in the network containing 3 to 500 components.

\section{High-performance liquid chromatography (HPLC) fingerprints of GXV}

HPLC fingerprints of GXV were performed as described previously $[25,56,57]$ with some modifications.

\section{Chromatographic conditions}

The separations were developed on a Diamonsil-C18 column $(4.6 \mathrm{~mm} \times 250 \mathrm{~mm}, 5 \mu \mathrm{m})$ with a constant temperature at $30{ }^{\circ} \mathrm{C}$. The mobile phases consisted of methanol (A) and $0.1 \%$ formic acid (B) using a gradient elution as follows: $0 \mathrm{~min} 5 \% \mathrm{~A}, 25 \mathrm{~min} 20 \% \mathrm{~A}, 30 \mathrm{~min}$ $40 \%$ A, $50 \min 60 \%$ A, $55 \min 95 \%$ A, 65 min 95\% A, 68 $\min 5 \% \mathrm{~A}, 70 \mathrm{~min} 5 \% \mathrm{~A}$, with a $1.0 \mathrm{~mL} / \mathrm{min}$ flow rate. The injection volume was set as $10 \mu \mathrm{L}$, and the detection wavelength was set at $270 \mathrm{~nm}$. All analyses were performed on an Agilent 1290 Infinity HPLC system (Agilent, Santa Clara, USA).

\section{Preparation of sample solutions}

Salvianolic Acid B (111562-201,212, National Institutes for Food and Drug Control), Salvianolic Acid A (120, 412, Shanghai Ronghe Pharmaceutical Technology Co., Ltd.), Salvianic Acid A Sodium (111366-201,305, National Institutes for Food and Drug Control), Paeoniflorin (110736-201,136, National Institutes for Food and Drug Control), Paeonol (110708-201,407, National Institutes for Food and Drug Control), and Rosmarinic Acid (20283-92-5, Nanjing Chunqiu Biological Engineering Co., Ltd.) were accurately weighed and added to methanol at concentrations of $0.1,0.05,0.1,0.1,0.1$, and 0.01 $\mathrm{mg} / \mathrm{mL}$ as the mixed reference solution.

One-fifth of the prescriptions of GXV (Codonopsis Radix (Origin Gansu, Anhui Songshantang Chinese Medicine Co., Ltd.) 40 g, Ophiopogon japonicus (Origin Sichuan, Bozhou Yonggang Pieces Factory Co., Ltd.) 20 g, Schizandra Chinensis Fructus (Origin Jilin, Bozhou Yonggang Pieces Factory Co., Ltd.) 10 g, Rehmannia Radix Praeparata (Origin He'nan, Bozhou Jingwan Traditional Chinese Medicine Pieces Factory) $40 \mathrm{~g}$, Radix Salviae (Origin Jiangsu, Anhui Huchuntang Chinese Herbal Pieces Co., Ltd.) 40 g, and Radix Paeoniae Rubra (Origin Neimenggu, Bozhou Jingwan Traditional Chinese Medicine Pieces Factory) 40 g,which were authenticated by the chief Chinese pharmacist and met the requirements of the 2015 Chinese Pharmacopoeia) was soaked in a tenfold volume of distilled water at room temperature for half an hour, decocted for $1.5 \mathrm{~h}$, filtered, and then an eightfold volume of distilled water was added into the filter residue for further decocting for an hour. All filtrates were vacuum concentrated as a GXV mixture. The amount of the $1 \mathrm{~mL}$ GXV mixture was accurately measured and extracted it with $9 \mathrm{ml}$ of methanol for half an hour. Before HPLC analysis, the volume loss during ultrasound was compensated, and then the extract was filtered by a $0.22 \mu \mathrm{m}$ membrane filter.

One-fifth of the prescriptions of Codonopsis Radix (Origin Gansu, Anhui Songshantang Chinese Medicine Co., Ltd.), Ophiopogon japonicus (Origin Sichuan, Bozhou Yonggang Pieces Factory Co., Ltd.), Schizandra Chinensis Fructus (Origin Jilin, Bozhou Yonggang Pieces Factory Co., Ltd.), Rehmannia Radix Praeparata (Origin He'nan, Bozhou Jingwan Traditional Chinese Medicine Pieces Factory), Radix Salviae (Origin Jiangsu, Anhui Huchuntang Chinese Herbal Pieces Co., Ltd.), and Radix Paeoniae Rubra (Origin Neimenggu, Bozhou Jingwan Traditional Chinese Medicine Pieces Factory), which were authenticated by the chief Chinese pharmacist and met the requirements of the 2015 Chinese Pharmacopoeia, were accurately weighed, and the preparations were tested according to the preparation method of GXV to obtain the crude drug solution.

\section{Method validation}

The precision, stability, and repeatability were assessed as described previously $[56,57]$ and expressed by the relative standard deviation of the average retention time and peak areas. Each sample solution was detected twice in parallel.

\section{Results}

Investigation of the active phytochemical compounds of GXV

The active phytochemical compounds contained in the six herbs (Codonopsis Radix, Ophiopogon japonicus, Schizandra Chinensis Fructus, Rehmannia Radix Praeparata, Radix Salviae, and Radix Paeoniae Rubra) that comprise GXV were identified from TCMSP, TCMID, and BATMAN-TCM. Consequently, by defining $\mathrm{OB}$ and DL, we obtained 21, 1, 8, 2, 65, and 29 compounds for Codonopsis Radix, Ophiopogon japonicus, Schizandra Chinensis Fructus, Rehmannia Radix Praeparata, Radix Salviae, and Radix Paeoniae Rubra, respectively. Some active compounds exist in many kinds of herbs (Fig. 1, Table S2), and 119 active compounds were identified after duplicate removal. To understand the multicomponent pharmacological mechanisms of GXV, we built an $\mathrm{H}-\mathrm{C}$ network (Fig. 2). The H-C network for GXV was composed of 126 nodes (including GXV, the six herbs, and 119 active compounds) and 132 edges (Fig. 2), indicating that there was much crossover between herbs and compounds. 


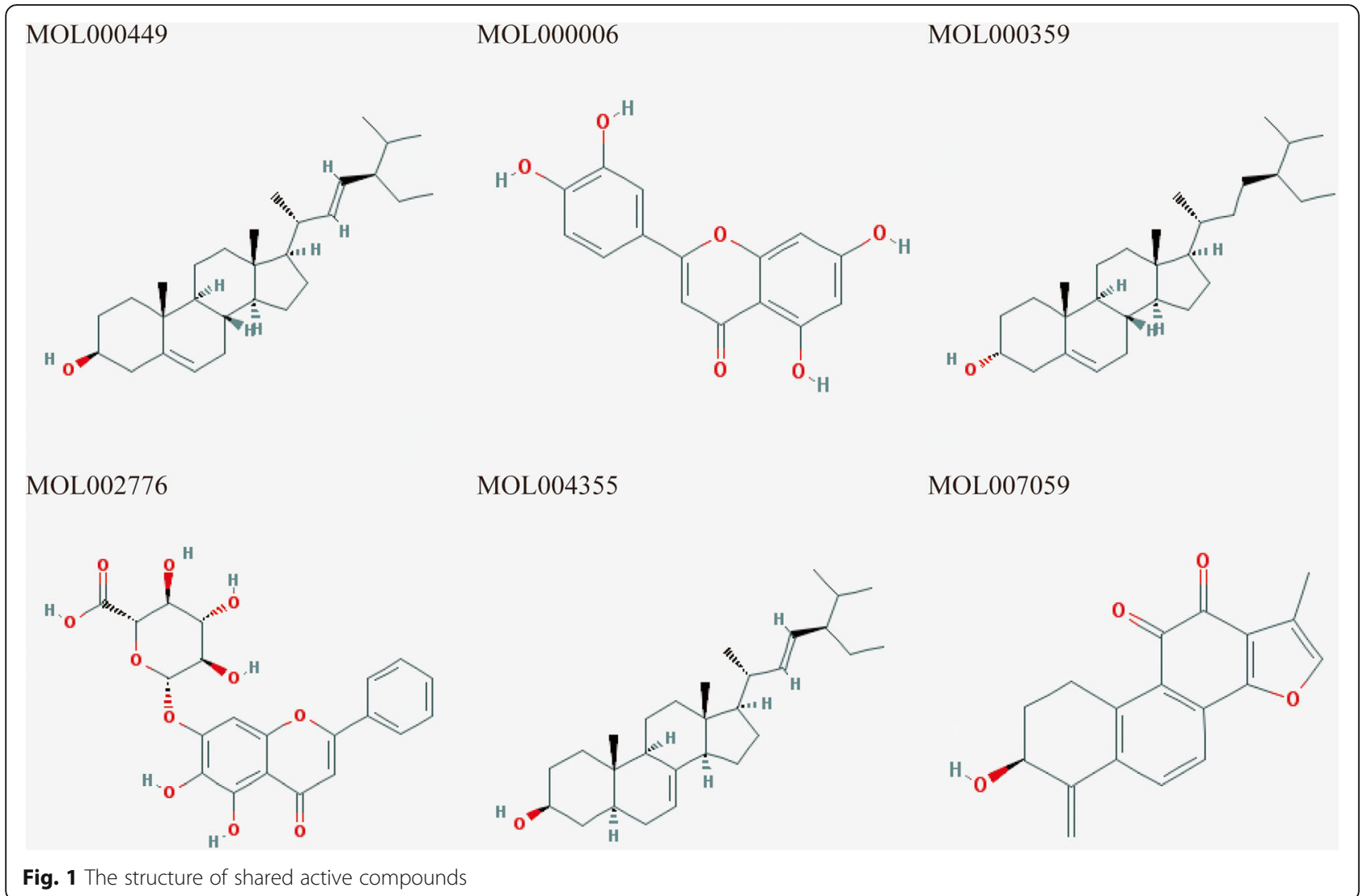

\section{Identification of the targets of active phytochemical compounds in GXV}

We obtained 1367 targets for the 119 active phytochemical compounds in GXV $(214,1,30,34,930$, and 158 compounds for Codonopsis Radix, Ophiopogon japonicus,
Schizandra Chinensis Fructus, Rehmannia Radix Praeparata, Radix Salviae, and Radix Paeoniae Rubra, respectively) by searching the corresponding database. Note that there were 181 targets after duplication removal, suggesting that different active compounds had the same targets.

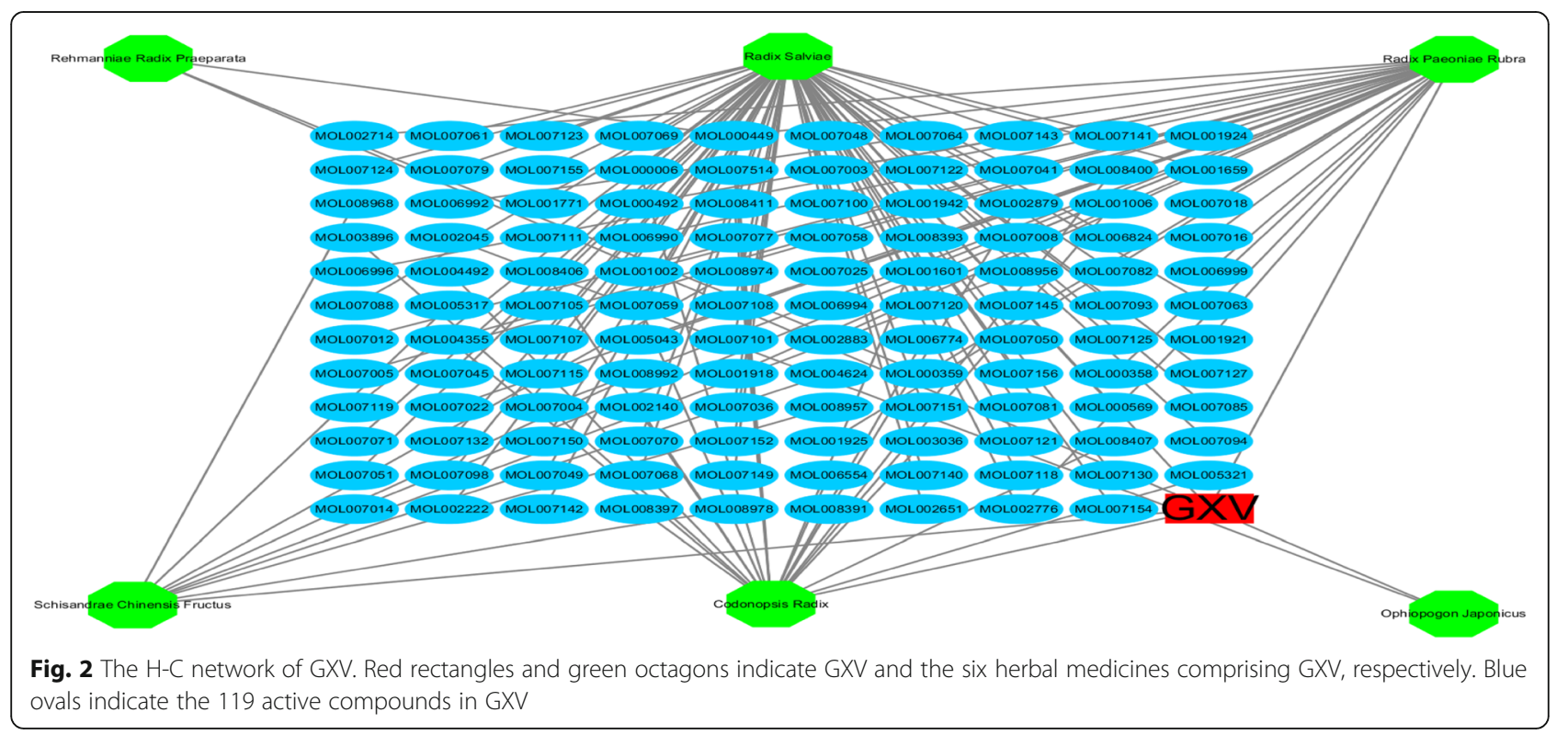


To further understand the multicomponent and multitarget mechanisms of GXV, an $\mathrm{H}-\mathrm{C}-\mathrm{T}$ network was constructed. This network for GXV was composed of 307 nodes (including GXV, the six herbs, 119 active compounds, and 181 targets) and 1499 edges (Fig. 3), investigating the system-level therapeutic properties of GXV.

\section{Identification of the targets of CAD}

To identify CAD-associated human targets, we surveyed diverse databases (GeneCards [37], TTD [38], DrugBank [39], DisGeNET [40, 41], OMIM, TCMSP [30], MalaCards [42], NCBI-Gene, and CTD [43]). Hence, a total of 2336 targets were obtained, with 2028 remaining after deduplication.

\section{Identification of shared targets of GXV and CAD}

After we uploaded 2028 CAD targets and 181 GXV targets, 2026 CAD targets and 181 GXV targets were identified as unique elements, and 2086 unique elements existed in total, which meant that 121 targets were shared by CAD and GXV.

\section{H-C-T-D network-based analysis of the pharmacological mechanisms of GXV}

The potential targets shared by the active compounds contained in GXV and CAD were applied for subsequent analysis. Because there were too many defined CAD targets, we only used the shared targets with GXV to build the H-C-T-D network diagram (Fig. 4). The H-C-T-D network was composed of 248 nodes (including CAD, GXV, the six herbal medicines, 119 active compounds, and 121 shared targets) and 1059 edges.

\section{Functional enrichment analysis}

The targets significantly associated with GXV and CAD that we uploaded were converted into their corresponding gene IDs with the latest version of the database (last updated on 2019-08-14). The overlaps between these targets are shown in a Circos plot [58] (Fig. 5a). Another useful representation is to overlap genes based on their functions or shared pathways. The overlaps between gene lists can be significantly improved by considering overlaps between genes sharing the same enriched ontology terms (Fig. 5b). Only ontology terms that contain

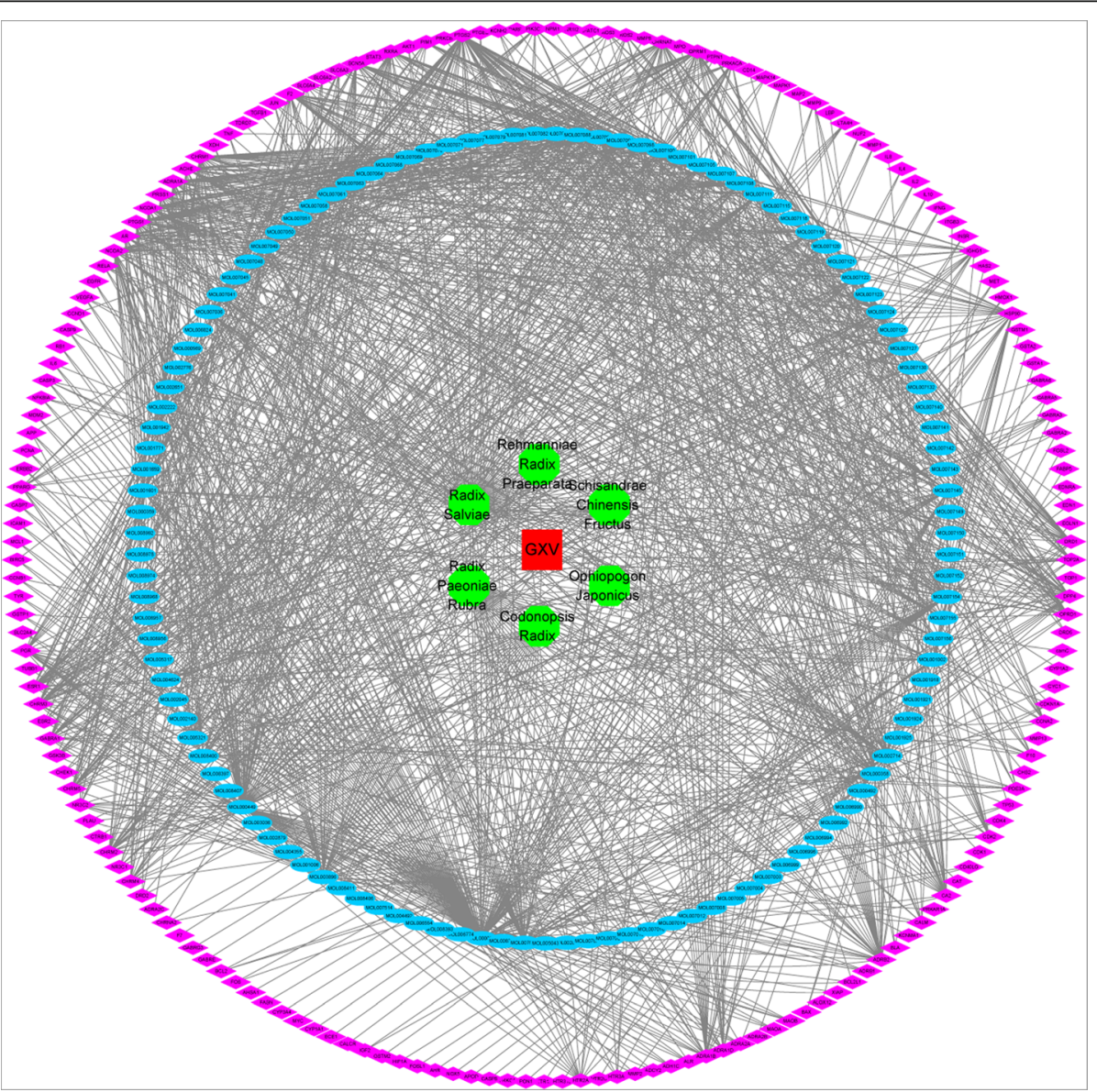

Fig. 3 The H-C-T network of GXV. Red rectangles and green octagons indicate GXV and the six herbal medicines comprising GXV, respectively. Blue ovals and purple diamonds indicate the 119 active compounds and 181 targets in GXV, respectively 


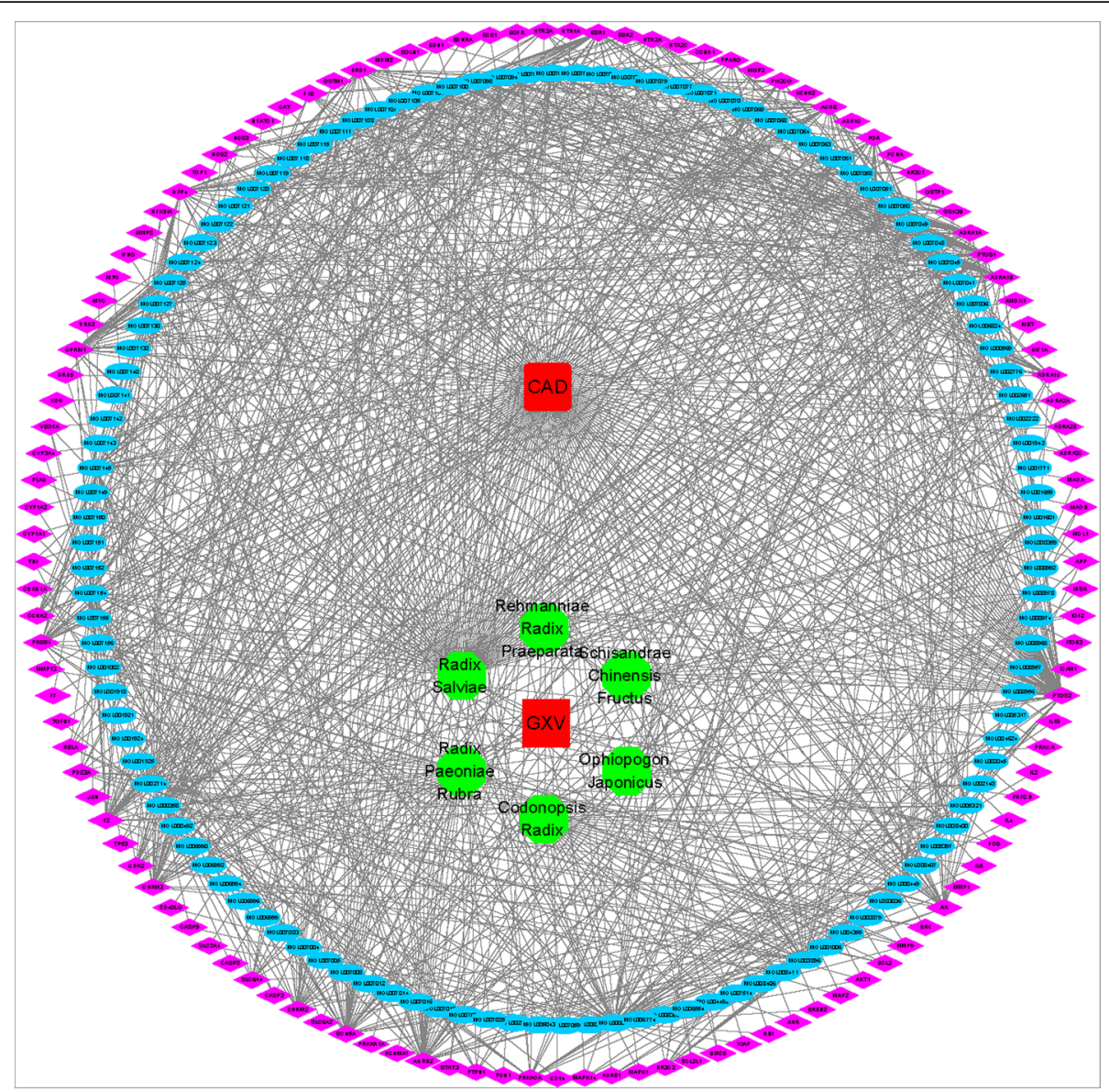

Fig. 4 The H-C-T-D network

less than 100 genes were used to calculate functional overlaps to avoid linking genes using very general annotation. From the heatmap of the top 20 enriched terms across targets significantly associated with GXV and CAD (Fig. 6), we found that the functions for these targets were mainly circulatory system and response, including blood vessel development, blood circulation, cytokine production, heart development, regulation of MAPK cascade, response to growth factor, positive regulation of cell death, signaling by interleukins, cellular response to nitrogen compound, wound healing, response to inorganic substance, cellular response to lipid, response to toxic substance, response to extracellular stimulus, extracellular structure organization, response to molecule of bacterial origin, response to oxygen levels, muscle cell proliferation, and vascular process in circulatory system.

The functions of shared targets were enriched by GO and KEGG from Metascape. The top 20 GO enrichment items were classified into three functional groups: biological process group (19 items), molecular function group (0 items), and cellular component group (1 item) (Fig. 7a). The network of GO enriched terms showed
167 nodes and 1439 edges (Fig. 7b). The shared 121 targets were mainly enriched in response, blood circulation, and apoptosis biological processes such as response to toxic substance, cytokine-mediated signaling pathway, blood circulation, response to inorganic substance, cellular response to nitrogen compound, cellular response to organic cyclic compound, response to wounding, regulation of secretion by cell, positive regulation of MAPK cascade, positive regulation of cellular component movement, apoptotic signaling pathway, response to oxygen levels, reactive oxygen species metabolic process, response to extracellular stimulus, response to lipopolysaccharide, cellular response to drug, regulation of DNAbinding transcription factor activity, second-messengermediated signaling, and response to radiation signaling pathway. The cellular components that these genes were involved in were membrane rafts. The top 20 KEGG pathways for the shared targets are shown in Fig. 7c. The network of KEGG enriched terms showed 112 nodes and 1098 edges (Fig. 7d). Among these pathways, the PI3K-Akt signaling pathway, HIF-1 signaling pathway, fluid shear stress and atherosclerosis, calcium signaling pathway, cAMP signaling pathway, serotonergic 


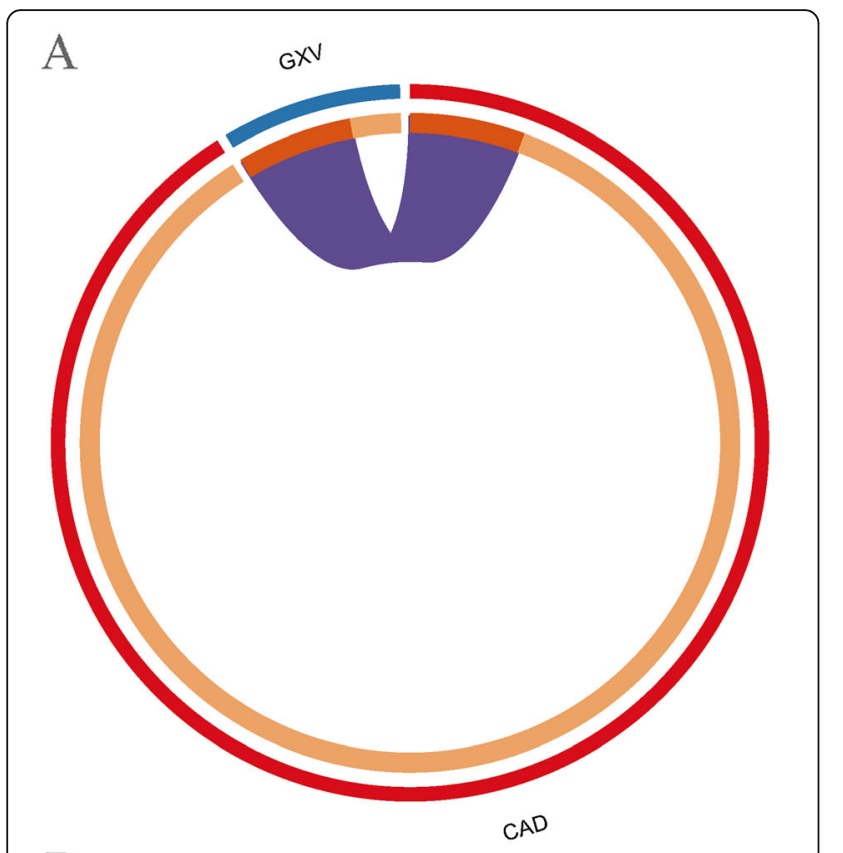

B

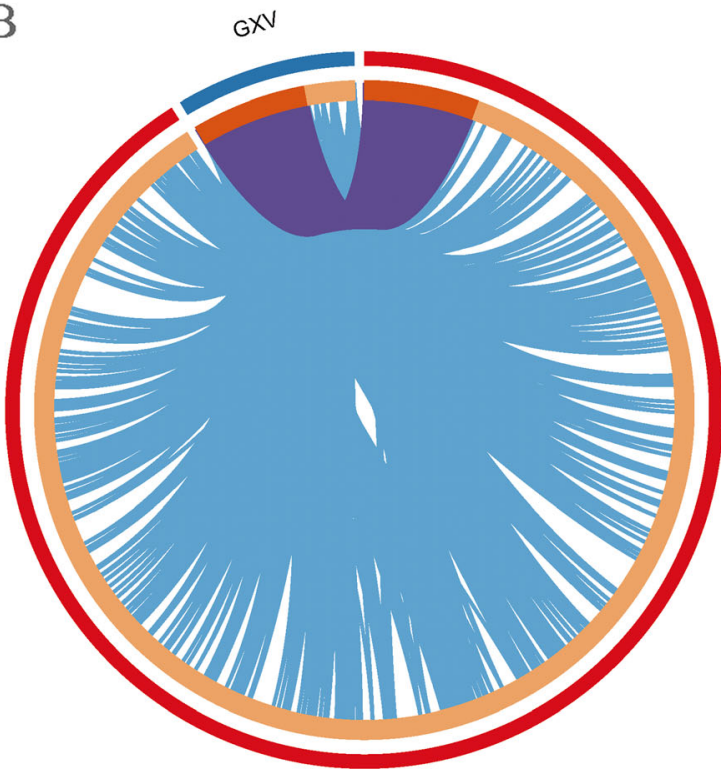

$C A D$

Fig. 5 Overlap between GXV and CAD targets. a Only at the gene level. On the outside, each arc represents the identity of each gene list. On the inside, each arc represents a gene list, where each gene has a spot on the arc. Dark orange represents the genes that appear in multiple lists and light orange represents genes that are unique to that gene list. Purple lines link the same genes that are shared by multiple gene lists. The greater the number of purple links and the longer the dark orange arcs, the greater overlap among the input gene lists. b Including the shared term level. Blue lines link the different genes where they fall into the same ontology term (the term has to be statistically significantly enriched and with a size no larger than 100). Blue links indicate the amount of functional overlap among the input gene lists synapse, thyroid hormone signaling pathway, regulation of lipolysis in adipocytes, and drug metabolismcytochrome P450 were found to be related to the development of multiple cardiovascular diseases and were involved in CAD development and pathogenesis. These findings support the pharmacological mechanisms of GXV in CAD.

In addition, to better understand the relationship between GXV targets and CAD, we performed a PPI enrichment analysis (Fig. 7e), which indicated 115 nodes and 635 edges. The MCODE networks identified for individual target lists have been gathered and are shown in Fig. 7f. The MCODE results showed that biological function was mainly related to the p53 signaling pathway, neuroactive ligand-receptor interaction, cGMP-PKG signaling pathway, cAMP signaling pathway, apoptosis, calcium signaling pathway, cAMP signaling pathway, neuroactive ligand-receptor interaction, and metabolism of xenobiotics by cytochrome P450.

\section{Specific chemical identification}

The Similarity Evaluation System for Chromatographic Fingerprints of TCMs Software (version 2004A) recommended by the China Food and Drug Administration was used for analysis. Fig. S1A shows the HPLC fingerprints of ten batches of GXV samples (S1-10). Sixteen characteristic common peaks (1-16) were automatically selected in the fingerprints. The relative standard deviation values of the average retention time and peak areas did not exceed 2 and 3\%, respectively, indicating that the method is good. The mixed reference solution was used to identify the characteristic common peaks. Six compounds were identified as Salvianolic Acid B (14), Salvianolic Acid A (15), Salvianic Acid A Sodium (5), Paeoniflorin (10), Paeonol (16), and Rosmarinic Acid (12) (Fig. S1B). Through comparison with the crude drug solution, it can be determined that peaks 1, 2, 3, 9, 10, and 16 were derived from Radix Paeoniae Rubra; peaks 5, 13, and 14 were derived from Codonopsis Radix; peaks 4 and 6 were derived from Schizandra Chinensis Fructus (Fig. S1C). The other five peaks (peaks 7, 8, 11, 12 , and 15) were unknown, which may be caused by cofried 6 herbs in the production process.

\section{Discussion}

TCM has a long history in clinical practice [59] and is gradually recognized at home and abroad [60]. CAD is the leading cause of mortality worldwide [61]. Great progress has been made in the treatment of CAD by TCM in recent years [62, 63]. Previous studies have shown that GXV combined with conventional medicine has a better curative effect than conventional medicine alone in CAD $[8,9]$. GXV can significantly improve the clinical symptoms $[11,12]$, reduce the incidence of angina 


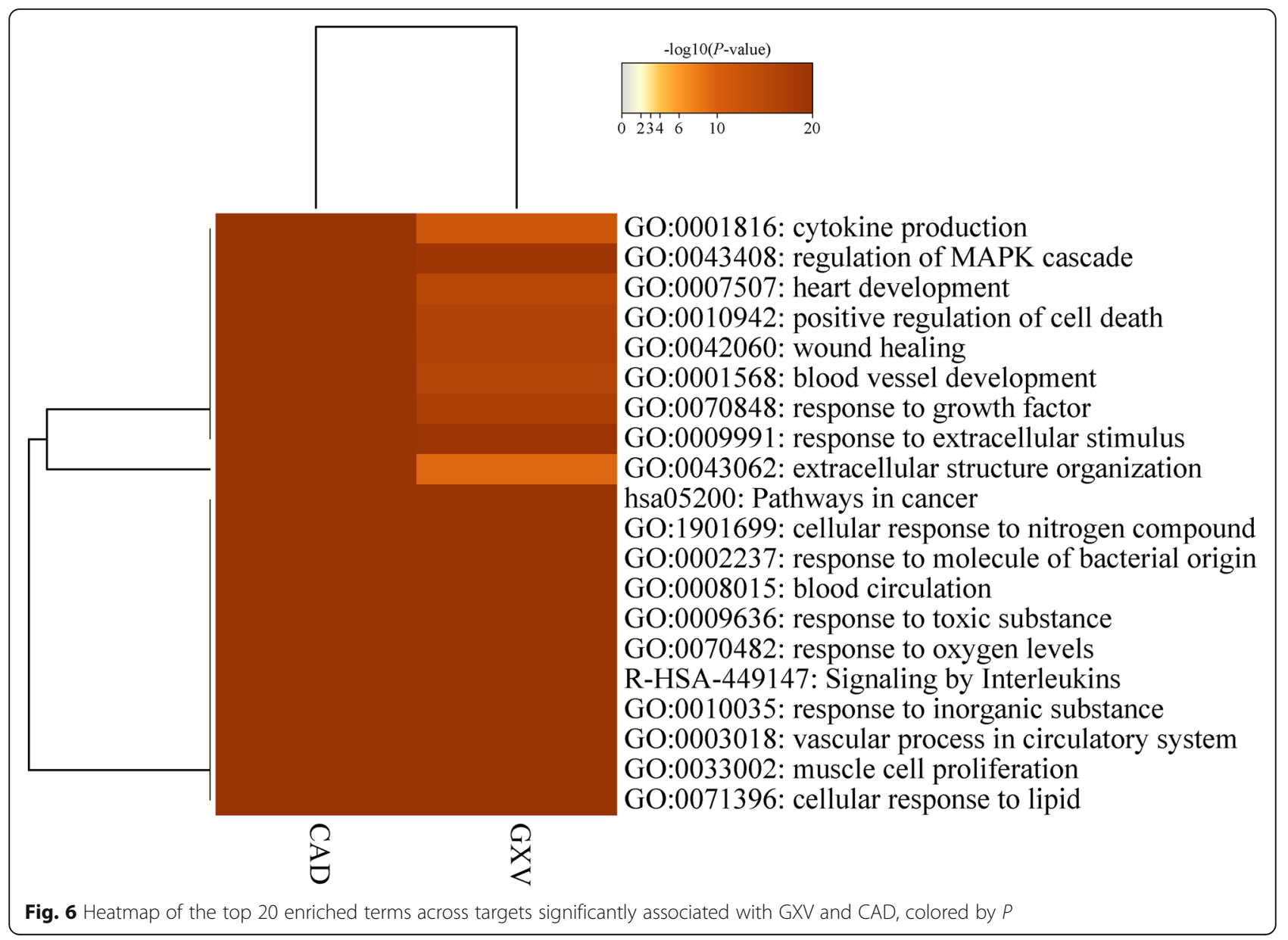

and the amount of nitroglycerin [11], improve the 24-h Holter myocardial ischemia load and heart rate variability [12], ejection fraction [10], stroke volume and cardiac output based on echocardiography [13]. Additionally, GXV reduces total cholesterol, triglycerides, and low density lipoprotein cholesterol [11], and increases high-density lipoprotein cholesterol [11] and six-minute walking distance [13]. The underlying mechanisms are also being explored. GXV lowers the serum levels of NT-pro BNP, hs-CRP, MMP-9, Ang II and ET-1 in patients with CAD [10].

In vivo experiments showed that GXV increases the ejection fraction and fractional shortening and reduces the left ventricular mass index $[14,15,18]$ and reduces the levels of IL- 6 , TNF- $\alpha$ and other inflammatory factors $[14,15]$ in rats with acute myocardial infarction by inhibiting the NF-kB pathway $[18,19]$.

Virtual screening and network pharmacology are effective ways to find the relationship between multiple components and targets of TCM $[64,65]$. In the present study, we identified the systemic mechanisms of GXV in the treatment of CAD by these approaches, which provides a new strategy to study the potential active components and targets of TCM [66, 67]. Our main findings can be summarized as follows: (I) 119 potentially active compounds from GXV had an interaction with 121 CAD-related targets, showing therapeutic activity; (II) functional enrichment analysis revealed that the targets from GXV were involved in various CAD-associated biological processes, such as cytokine-mediated signaling pathway, blood circulation, cellular response to nitrogen compound, response to wounding, regulation of secretion by cell, positive regulation of MAPK cascade, positive regulation of cellular component movement, apoptotic signaling pathway, response to oxygen levels, reactive oxygen species metabolic process, response to extracellular stimulus, response to lipopolysaccharide, cellular response to drug, and regulation of DNAbinding transcription factor activity; (III) the CADassociated targets of GXV were significantly enriched in diverse pathways, including the PI3K-Akt signaling pathway, HIF-1 signaling pathway, fluid shear stress and atherosclerosis, calcium signaling pathway, cAMP signaling pathway, serotonergic synapse, thyroid hormone signaling pathway, regulation of lipolysis in adipocytes, and drug metabolism-cytochrome P450, which are associated with CAD. 


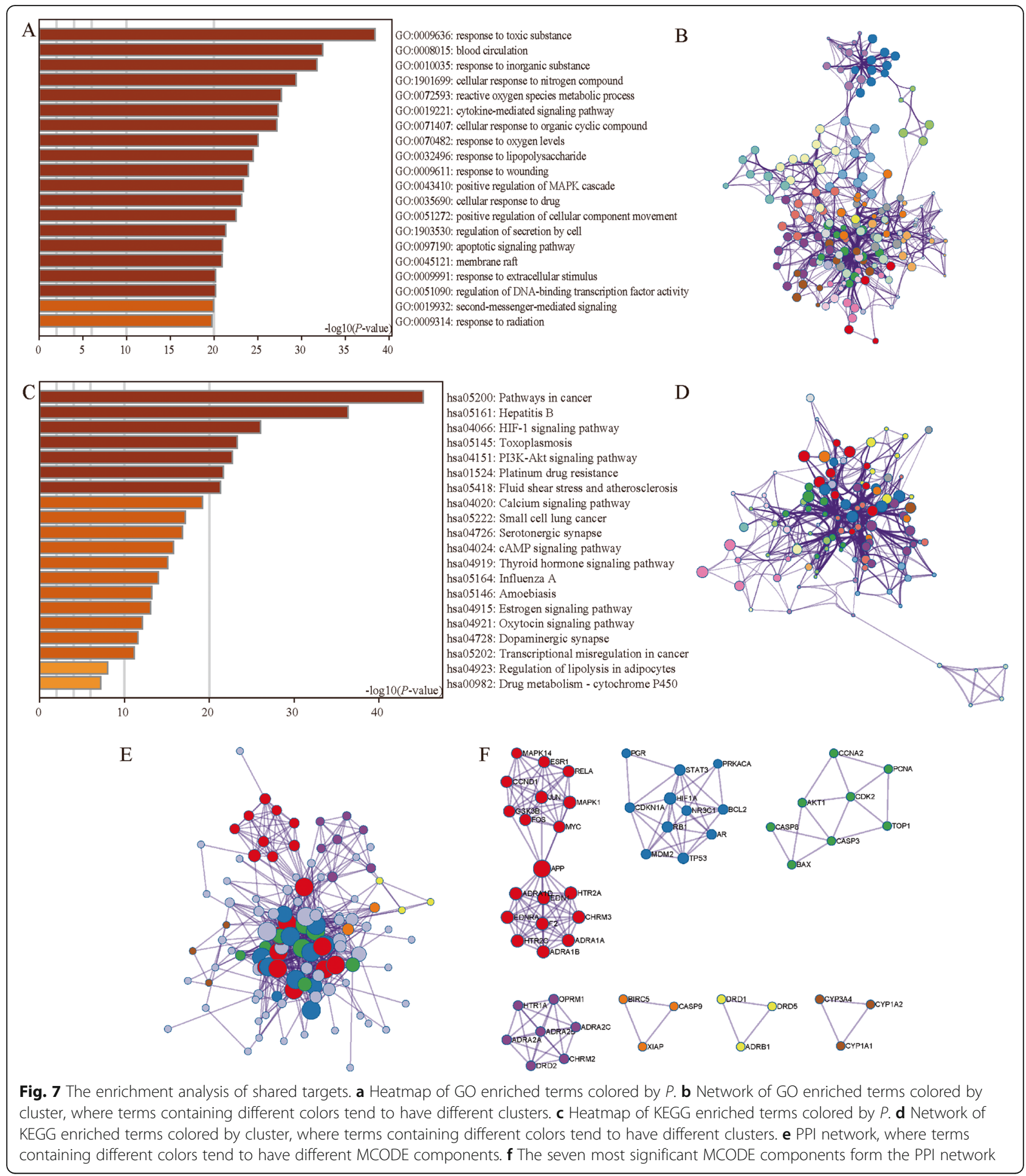

GXV is composed of six herbal medicines containing 119 active compounds that interact with 121 CAD-related targets. These herbs and chemical constituents of GXV have been reported to be beneficial to CAD. Codonopsis Radix shares immunomodulation effects [68, 69]. Radix Codonopsis polysaccharide, an active compound in Codonopsis
Radix, could maintain the T-cell balance against hydrocortisone disturbance [70]. Choushenpilosulynes A-C, isolated from Codonopsis Radix, can inhibit the expression of SQLE involved in lipid metabolism [71]. ShenMai injection, prepared from Panax ginseng and Ophiopogon japonicus, is used as an add-on therapy for CAD [72, 73]. The main 
components of Ophiopogon japonicus exhibit various pharmacological activities, such as cardiovascular protection [74-77], anti-inflammation [74, 76, 78-80], antioxidation [74-76, 81], mitochondrial function preservation [81], apoptosis inhibition [75, 81], and immunomodulation $[74,77]$. The extract of Ophiopogon japonicus decreased ICAM-1 and VCAM-1 to play an endothelial protective role from oxidative damage and dysfunction [82]. In addition, it also inhibited proliferation [82]. Ophiopogon japonicus has a regulatory impact on the cAMP signaling pathway, WNT signaling pathway, and PI3K-AKT signaling pathway by targeting HSPA8, TP53, and VEGFA [83]. The key cardioprotective mechanisms of Schizandra Chinensis Fructus and its active ingredients have been demonstrated to include anti-oxidation [84-87], suppression of apoptosis [84, 88], and anti-inflammation [84, 86, 87]. Schizandra Chinensis Fructus increases antioxidant capacity and improves endothelial dysfunction to ameliorate the extent of atherosclerosis [85]. Schizandra Chinensis Fructus extracts induce apoptosis via the ROS-mediated/mitochondriadependent pathway and JNK/p38 MAPK activation [89]. In addition, Schizandra Chinensis Fructus was found to facilitate PI3K-AKT activation and inhibit the expression of NOX2 in AMI mice and oxygen-glucose deprivationtreated H9c2 cells [90]. Rehmannia Radix Praeparata had efficient detectable antioxidant activity [91], and the PI3KAkt and MAPK signaling pathways were found in the pathway analysis for CAD on Rehmannia Radix Praeparata [92]. In addition, extraction from Rehmannia Radix Praeparata regulated the IGF-1/PI3K/mTOR signaling pathways [93]. The compounds from Radix Salviae showed various pharmacological activities, such as antiinflammation [94-96], antioxidation [94, 96, 97], direct thrombin inhibitory effects with a dose-effect relationship [98], antiproliferation [99], improvement in microcirculatory disturbances [96], blocking of calcium inflow and prevention of calcium overload [96], and anti-atherogenesis [94], and its mechanisms may be related to activating the Nrf2 pathway [97] and NF-kB modulation [95]. Radix Paeoniae Rubra has antiinflammatory [100], antiproliferation [101], antiapoptosis [102], immunoregulatory [100], scavenging free radicals [103], regulation of lipid metabolism [104], antifibrosis [103], and myocardial protection [102] properties via the NF-kB [100], MAPK [100], PI3K/ Akt/mTOR [102], and TGF- $\beta /$ Smad [103] signaling pathways. Moreover, Radix Paeoniae Rubra extract had an inhibitory effect on thrombus formation, and the antithrombotic effects were associated with the regulation of vascular endothelium active substances, activating blood flow and anticoagulation effects [105].

Fingerprints can comprehensively reflect the types and quantities of chemicals contained in medicines, thereby effectively evaluating and controlling the quality of TCM [106]. In this study, HPLC was used to establish the fingerprints of GXV, which can reflect the quality of the overall characteristics and provide a basis for overall quality control, thereby improving the stability and ensuring the safety and effectiveness of clinical medication. It also laid the foundation for basic research on the medicinal substances of GXV.

In conclusion, these previous findings support the potential role of herbal and chemical constituents of GXV in the treatment of CAD. Furthermore, we have validated the new potential therapeutic targets and underlying molecular mechanisms of GXV against CAD, which might provide a reference for its future application in cardiovascular diseases [29, 107, 108]. More studies are needed to further validate the therapeutic properties of GXV.

The results presented in this study improved our understanding of GXV, which is prescribed for CAD. The system mechanisms of GXV for CAD were identified through 119 major active ingredients and 121 candidate targets. In particular, those candidate targets were highly correlated with CAD in our functional enrichment results. These studies indicate the feasibility of the predicted biological processes and pathways. However, the regulation of GXV on key biological processes and key pathways in CAD needs further basic and clinical research confirmation. The findings of potential key targets may provide new clues for CAD treatments with GXV.

\section{Conclusions}

Via the method of integrative virtual screening and network pharmacology, our study predicts the targets of the ingredients of GXV and explores the underlying mechanisms of the potential anti-CAD effects, providing a complementary and alternative therapy for CAD. We have reasons to believe that the potential mechanisms are direct or indirect synergy of multitarget and multipathway efforts. However, more experimental validation is essential to reveal the effect of GXV against CAD.

\section{Supplementary Information}

The online version contains supplementary material available at https://doi. org/10.1186/s12906-020-03133-w.

\footnotetext{
Additional file $\mathbf{1}$ Table $\mathbf{S 1}$. Gene annotations extracted.

Additional file $\mathbf{2}$ Table S2. Details of compounds in various herbs.

Additional file $\mathbf{3}$ Fig. S1. The HPLC fingerprints of GXV. A) The fingerprints of 10 batches of GXV. B) The fingerprints of GXV (above) and mixed reference solution (below). C) The fingerprints of crude drugs.
}

\footnotetext{
Abbreviations

CAD: Coronary artery disease; DL: Drug likeness; GO: Gene Ontology;

GXV: Guanxin V; H-C: herb-compound; H-C-T: Herb-compound-target; H-C-TD: Herb-compound-target-disease; HPLC: High performance liquid

chromatography; KEGG: Kyoto Encyclopedia of Genes and Genomes; OB: Oral
} 
bioavailability; PPI: Protein-protein interaction; TCM: Traditional Chinese medicine; UniProt: Universal Protein

\section{Acknowledgements}

We are grateful to all research scientists who participated in the aforementioned databases. We thank Dr. Fu-Qiong Zhou from Nanjing Hospital of Chinese Medicine Affiliated to Nanjing University of Chinese Medicine for her excellent technical assistance about HPLC fingerprints. We thank Professor Wen Li from Nanjing Hospital of Chinese Medicine Affiliated to Nanjing University of Chinese Medicine for the authentication of all Chinese herbs. We also thank all individuals involved GXV.

\section{Authors' contributions}

$B L$ and NG conceived, designed, and planned the study. $B L$ and $X X Z$ acquired and analyzed the data. BL, NG and XXZ interpreted the results. BL drafted the manuscript and NG contributed to critical revision of the manuscript. All authors read and approved the final manuscript.

\section{Funding}

This work was funded by the National Natural Science Foundation of China [81774229], Jiangsu Universities Nursing Advantage Discipline Project [2019YSHL095], Major Project of Nanjing Medical Science and Technology Development During 13th Five-year Plan [ZDX16013], and Jiangsu Leading Talent Project of Traditional Chinese Medicine [Jiangsu TCM 2018 No.4].

\section{Availability of data and materials}

All data generated or analysed during this study are included in this published article and its supplementary information files.

\section{Ethics approval and consent to participate}

Not applicable.

\section{Consent for publication}

Not applicable.

\section{Competing interests}

Not applicable.

\section{Author details}

${ }^{1}$ Nanjing University of Chinese Medicine, Nanjing, China. ${ }^{2}$ Nanjing Hospital of Chinese Medicine Affiliated to Nanjing University of Chinese Medicine, Nanjing, China.

Received: 11 May 2020 Accepted: 26 October 2020

Published online: 13 November 2020

\section{References}

1. Zheng $X$, Curtis JP, Hu S, et al. Coronary catheterization and percutaneous coronary intervention in China: 10-year results from the China PEACEretrospective CathPCI study. JAMA Intern Med. 2016;176(4):512-21.

2. Dong Z, Liu J, Wang $M$, et al. Epidemiology of cardiovascular disease in China: current features and implications. Nat Rev Cardiol. 2019;16(4):203-12.

3. G. B. D. Causes of Death Collaborators. Global, regional, and national agesex-specific mortality for 282 causes of death in 195 countries and territories, 1980-2017: a systematic analysis for the global burden of disease study 2017. Lancet. 2018;392(10159):1736-88.

4. Liu S-W, Li Y-C, Zeng X-Y, et al. Burden of cardiovascular diseases in China, 1990-2016: findings from the 2016 global burden of disease study. JAMA Cardiol. 2019;4(4):342-52.

5. Shen C-X, Ge J-B. Epidemic of cardiovascular disease in China. Circulation. 2018;138(4):342-4

6. Knuuti J, Wijns W, Saraste A, et al. 2019 ESC guidelines for the diagnosis and management of chronic coronary syndromes. Eur Heart J. 2019;41(3):407-77.

7. Liang B, Yuan Q, Zhao Q-F, et al. Guanxin V for coronary artery disease: A retrospective study. Biomed Pharmacother. 2020;128:110280.

8. Shao Z-X, Ning G. Guanxin V in the treatment of stable angina of coronary heart disease: 46 case series. Jiangxi J Traditional Chin Med. 2008;39(311): $18-9$.
9. Shi A-W, Ning G, Wang J, et al. Guanxin V for coronary heart disease with deficiency of qi and yin with stasis syndrome. Henan Traditional Chin Med. 2008;27(07):48-50

10. Jun-Gong, Jiang W-B, Ning G. Clinical efficacy of Guanxin V in treating ventricular remodeling after acute myocardial infarction and its mechanism. China Pharmaceuticals. 2017;26(22):30-3.

11. Wang J. Combination of Chinese and conventional medicine in the treatment of stable angina of coronary heart disease with deficiency of qi and yin with stasis syndrome: 30 case series. Jiangsu J Trad Chin Med. 2013; 45(06):11-3.

12. Wei-Wan T-QW, Pang J-H. Combination of Chinese and conventional medicine in the treatment of stable angina pectoris of coronary heart disease: 30 case series. Jiangsu J Trad Chin Med. 2012;44(12):21-3.

13. Yuan $Q$, Ning G, Zhou J-Z. The effect of Guanxin- $V$ mixture with conventional medicine on heart function of coronary heart disease in patients with congestive heart failure. Shanghai Journal of Traditional Chinese Medicine. 2011;45(10):35-6.

14. Ke F, Zuo K-K, Gu N. Effect of Guanxin V on Ventricular Remodeling and Inflammatory Factor in Rats with Acute Myocardial Infarction. J Emerg Traditional Chinese Med. 2014;23(11):1997-9 +2033.

15. Ke F, Zuo K-K, Ning G. Effects of Guanxin V on rats ventricular remodeling and inflammatory factors with acute myocardial infarction. Journal of Basic Chinese Medicine. 2016;22(01):50-3.

16. Ke F, Zuo K-K, Ning G. Research progress of Guanxin $V$ in relieving ventricular remodeling after acute myocardial infarction. Chinese Journal of Integrative Medicine on Cardio-/Cerebrovascular Disease. 2015;13(11): 1289-91.

17. Ke F, Zuo K-K, Ning G. Research Progress on mechanisms of Chinese herbal compound interfere ventricular remodeling after acute myocardial infarction. Chin J Exp Tradit Med Formulae. 2014;20(16):234-6.

18. Zuo K-K, Ke F, Ning G. Effect and mechanism of Guanxin V on Ventriculan remodeling after acute myocardial infarction. Chin J Exp Tradit Med Formulae. 2015;21(05):147-50.

19. Zuo K-K, Ke F, Gu N. Guanxin V Mixture Intervention on the Ventricular Remodeling and Myocardial TLR4 Signal Pathway in Rat Model of Acute Myocardial Infarction. Journal of Basic Chinese Med. 2016;22(02):181-3 +194

20. Li S, Zhang B. Traditional Chinese medicine network pharmacology: theory, methodology and application. Chin J Nat Med. 2013;11(2):110-20.

21. Zhang G-B, Li Q-Y, Chen Q-L, et al. Network pharmacology: a new approach for chinese herbal medicine research. Evidence-based Complementary and Alternative Medicine. 2013;2013:621423.

22. Hopkins AL. Network pharmacology: the next paradigm in drug discovery. Nat Chem Biol. 2008;4(11):682-90.

23. Poornima P, Kumar JD, Zhao Q, et al. Network pharmacology of cancer: from understanding of complex interactomes to the design of multi-target specific therapeutics from nature. Pharmacol Res. 2016;111:290-302.

24. Lee H-S, Lee I-H, Park S-I, et al. Network Pharmacology-Based Investigation of the System-Level Molecular Mechanisms of the Hematopoietic Activity of Samul-Tang, a Traditional Korean Herbal Formula. Evidence-based Complementary and Alternative Medicine. 2020;2020:9048089.

25. Liang B, Zhou Y, Ling F, et al. Antiarrhythmic Mechanisms of Chinese Herbal Medicine Dingji Fumai Decoction. Evidence-Based Complementary and Alternative Medicine. 2020;2020:9185707.

26. Liang B, Zou F-H, Ling F, et al. Chinese Herbal Medicine Dingji Fumai Decoction for Ventricular Premature Contraction: A Real-World Trial. BioMed Res Int. 2020;2020:5358467.

27. Liang B, Luo F-Y, Liao H-L. Study on the mechanism of antihypertensive and synergistic effect of wind drugs on hypertensive rats with liver-yang hyperactivity based on vasoactive substances. Basic Clin Pharmacol Toxicol. 2018;124:32.

28. Cen W, Chen Z-L, Ning G, et al. Prevention of AMI Induced Ventricular Remodeling: Inhibitory Effects of Heart-Protecting Musk Pill on IL-6 and TNF-Alpha. Evidence-based Complementary and Alternative Med. 2017; 2017:3217395.

29. Zhu Y, Zhang Y-J, Huang $X$, et al. Z-Ligustilide protects vascular endothelial cells from oxidative stress and rescues high fat diet-induced atherosclerosis by activating multiple NRF2 downstream genes. Atherosclerosis. 2019;284: $110-20$.

30. Jin-Long R, Li P, Wang J, et al. TCMSP: a database of systems pharmacology for drug discovery from herbal medicines. J Cheminformatics. 2014;6:13. 
31. Xue R-C, Fang Z, Zhang M-X, et al. TCMID: traditional Chinese medicine integrative database for herb molecular mechanism analysis. Nucleic Acids Res. 2012;41(D1):D1089-95.

32. Liu Z-Y, Guo F-F, Wang Y, et al. BATMAN-TCM: a Bioinformatics Analysis Tool for Molecular mechANism of Traditional Chinese Medicine. Scientific Reports. 2016;6:21146.

33. Wang CK, Craik DJ. Cyclic peptide oral bioavailability: lessons from the past. Biopolymers. 2016;106(6):901-9.

34. Yeong Lee A, Park W, Kang T-W, et al. Network pharmacology-based prediction of active compounds and molecular targets in Yijin-Tang acting on hyperlipidaemia and atherosclerosis. J Ethnopharmacol. 2018;221:151-9.

35. Song W-J, Ni S-L, Yan-Ling F, et al. Uncovering the mechanism of maxing Ganshi decoction on asthma from a systematic perspective: a network pharmacology study. Sci Rep. 2018;8(1):17362.

36. Zhang J, Zheng C-L, Yuan S-Y, et al. Uncovering the Pharmacological Mechanism of Decoction on Epilepsy by Network Pharmacology Analysis. Evidence-based Complementary and Alternative Med. 2019;2019:3104741.

37. Safran M, Dalah I, Alexander J, et al. GeneCards Version 3: the human gene integrator. Database. 2010;2010(1):baq020.

38. Wang Y-X, Zhang S, Li F-C, et al. Therapeutic target database 2020: enriched resource for facilitating research and early development of targeted therapeutics. Nucleic Acids Res. 2020;48(D1):D1031-41.

39. Wishart DS, Feunang YD, Guo AC, et al. DrugBank 5.0: a major update to the DrugBank database for 2018. Nucleic Acids Res. 2018;46(D1):D1074-82.

40. Piñero J, Bravo À, Queralt-Rosinach N, et al. DisGeNET: a comprehensive platform integrating information on human disease-associated genes and variants. Nucleic Acids Res. 2017;45(D1):D833-9.

41. Piñero J, Ramírez-Anguita JM, Saüch-Pitarch J, et al. The DisGeNET knowledge platform for disease genomics: 2019 update. Nucleic Acids Res. 2020;48(D1):D845-55.

42. Rappaport N, Twik M, Plaschkes I, et al. MalaCards: an amalgamated human disease compendium with diverse clinical and genetic annotation and structured search. Nucleic Acids Res. 2017;45(D1):877-87.

43. Davis AP, Grondin CJ, Johnson RJ, et al. The comparative Toxicogenomics database: update 2019. Nucleic Acids Res. 2018;41(D1):948-54.

44. Barabási A-L, Oltvai ZN. Network biology: understanding the cell's functional organization. Nat Rev Genet. 2004;5(2):101-13.

45. The UniProt Consortium. UniProt: a worldwide hub of protein knowledge. Nucleic Acids Res. 2018;47(D1):D506-15.

46. Shannon P, Markiel A, Ozier O, et al. Cytoscape: a software environment for integrated models of biomolecular interaction networks. Genome Res. 2003; 13(11):2498-504.

47. Zhou Y-Y, Zhou B, Pache L, et al. Metascape provides a biologistoriented resource for the analysis of systems-level datasets. Nat Commun. 2019;10(1):1523.

48. Ashburner M, Ball CA, Blake JA, et al. Gene ontology: tool for the unification of biology. The gene ontology consortium. Nat Genet. 2000;25(1):25-9.

49. Kanehisa M, Goto S. KEGG: Kyoto Encyclopaedia of genes and genomes. Nucleic Acids Res. 2000;28(D1):D27-30.

50. Zhou J-G, Liang B, Jin S-H, et al. Development and Validation of an RNASeq-Based Prognostic Signature in Neuroblastoma. Frontiers Oncol. 2019;9:1361

51. Zhou Q, Zhang F, He Z, et al. E2F2/5/8 Serve as Potential Prognostic Biomarkers and Targets for Human Ovarian Cancer. Frontiers Oncol. 2019;9:161.

52. Oughtred R, Stark C, Breitkreutz B-J, et al. The BioGRID interaction database: 2019 update. Nucleic Acids Res. 2019;47(D1):D529-41.

53. Li T-B, Wernersson R, Hansen RB, et al. A scored human protein-protein interaction network to catalyze genomic interpretation. Nat Methods. 2017; 14(1):61-4.

54. Türei D, Korcsmáros T, Saez-Rodriguez J. OmniPath: guidelines and gateway for literature-curated signaling pathway resources. Nat Methods. 2016;13(12): 966-7.

55. Gary D, Bader CW. V Hogue An automated method for finding molecular complexes in large protein interaction networks. BMC Bioinformatics. 2003:4:2.

56. Guo L, Gong M-X, Wu S, et al. Identification and quantification of the quality markers and anti-migraine active components in Chuanxiong Rhizoma and Cyperi Rhizoma herbal pair based on chemometric analysis between chemical constituents and pharmacological effects. J Ethnopharmacol. 2020; 246:112228.
57. Zhou F-Q, Teng L-X, Yu L, et al. Elaboration of the comprehensive metabolic profile of Salvianolic acid a in vivo and in vitro using UFLC-Q/TOF-MS. J Agric Food Chem. 2019;67(44):12199-207.

58. Krzywinski M, Schein J, Birol I, et al. Circos: an information aesthetic for comparative genomics. Genome Res. 2009;19(9):1639-45.

59. Chao J, Dai Y-T, Verpoorte R, et al. Major achievements of evidence-based traditional Chinese medicine in treating major diseases. Biochem Pharmacol. 2017;139:94-104.

60. Liang $B$, Jing $M-T$, Liao $H-L$. Research and prospective of traditional Chinese medicine on sodium ion channels. Drug Combination Therap. 2019;1(1):2-8.

61. Chen K-B, Chen K-C, Chang Y-L, et al. In Silico investigation of traditional Chinese medicine for potential Lead compounds as SPG7 inhibitors against coronary artery disease. Molecules. 2016;21(5):588.

62. Hao P-P, Jiang F, Chen Y-G, et al. Traditional Chinese medication for cardiovascular disease. Nat Rev Cardiol. 2015:12(2):115-22.

63. Hao P-P, Jiang F, Cheng J, et al. Traditional Chinese medicine for cardiovascular disease: evidence and potential mechanisms. J Am Coll Cardiol. 2017;69(24):2952-66.

64. Ma X-Q, Yu M-X, Hao C-X, et al. Identifying Synergistic Mechanisms of Multiple Ingredients in Shuangbai Tablets against Proteinuria by Virtual Screening and a Network Pharmacology Approach. Evidence-based Complementary and Alternative Med. 2020;2020:1027271.

65. Wan Y-X, Xu L, Ze-Yu L, et al. Utilising network pharmacology to explore the underlying mechanism of Wumei pill in treating pancreatic neoplasms. BMC Complement Altern Med. 2019;19(1):158.

66. Liu C-x, Liu R, Fan H-R, et al. Network pharmacology bridges traditional application and modern development of traditional Chinese medicine. Chin Herbal Med. 2015;7(1):3-17.

67. Zhang Y-Q, Mao X, Guo Q-Y, et al. Network pharmacology-based approaches capture essence of Chinese herbal medicines. Chin Herbal Med. 2016;8(2):107-16.

68. Liu C-S, Wang Y-P, Shi Y-B, et al. Effect of Codonopsis Radix maintained with sulfur fumigation on immune function in mice. Zhong Yao Cai. 2014;37(11): 1969-72.

69. Zou Y-F, Zhang Y-Y, Yu-Ping F, et al. A polysaccharide isolated from with immunomodulation effects both in vitro and in vivo. Molecules. 2019;24(20):3632.

70. Deng X-L, Ya-Jun F, Luo S, et al. Polysaccharide from Radix Codonopsis has beneficial effects on the maintenance of T-cell balance in mice. Biomedicine and Pharmacother. 2019;112:108682.

71. Hu X-Y, Qin F-Y, Lu X-F, et al. Three new Polyynes from Codonopsis pilosula and their activities on lipid metabolism. Molecules. 2018;23(4):887.

72. Olaleye $\mathrm{OE}$, Niu W, Fei-Fei $\mathrm{D}$, et al. Multiple circulating saponins from intravenous ShenMai inhibit OATP1Bs in vitro: potential joint precipitants of drug interactions. Acta Pharmacol Sin. 2019;40(6):833-49.

73. Zhang C-X, Zheng Y-G, Chen T, et al. The utility of traditional Chinese medicine (Shenmai) in the cardiac rehabilitation after coronary artery bypass grafting: A single-center randomized clinical trial. Complementary Therapies Med. 2019;47:102203.

74. Chen M-H, Chen X-J, Wang M, et al. Ophiopogon japonicus--a phytochemical, ethnomedicinal and pharmacological review. J Ethnopharmacol. 2016:181:193-213.

75. Wang L-L, Zhou Y-F, Qin Y-C, et al. Methylophiopogonanone B of Radix Ophiopogonis protects cells from $\mathrm{H} 2 \mathrm{O} 2$-induced apoptosis through the NADPH oxidase pathway in HUVECs. Mol Med Rep. 2019;20(4):3691-700.

76. Wu Z-W, Zhao X-K, Miyamoto A, et al. Effects of steroidal saponins extract from Ophiopogon japonicus root ameliorates doxorubicin-induced chronic heart failure by inhibiting oxidative stress and inflammatory response. Pharm Biol. 2019;57(1):176-83.

77. Liu H-J, Wang Y, Wang T-Z, et al. De novo assembly and annotation of the Zhe-Maidong (Ophiopogon japonicus (L.f.) Ker-Gawl) transcriptome in different growth stages. Scientific Rep. 2017;7(1):3616.

78. Yao Q-W, Wang X-Y, Li J-C, et al. Ophiopogon japonicus inhibits radiationinduced pulmonary inflammation in mice. Ann Transl Med. 2019;7(22):622.

79. Kitahiro $Y$, Koike A, Sonoki A, et al. Anti-inflammatory activities of Ophiopogonis Radix on hydrogen peroxide-induced cellular senescence of normal human dermal fibroblasts. J Nat Med. 2018;72(4):905-14.

80. Dang NH, Chung ND, Tuan HM, et al. 2-Benzyl-benzofurans from the tubers of Ophiopogon japonicus. Chemistry Central J. 2017;11:-15.

81. Qian J-Y, Deng P, Liang Y-D, et al. 8-Formylophiopogonanone Antagonizes Paraquat-Induced Hepatotoxicity by Suppressing Oxidative Stress. Frontiers Pharmacol. 2019;10:1283. 
82. Tian Y-S, Gong P-Y, Wu Y, et al. Screening and identification of potential active components in Ophiopogonis Radix against atherosclerosis by biospecific cell extraction. J Chromatography B. 2019;1133:121817.

83. Feng $X-S$, Shi H-L, Xu C, et al. Deciphering the Pharmacological Mechanism of the Herb in the Treatment of Nasopharyngeal Carcinoma by Integrating iTRAQ-Coupled 2-D LC-MS/MS Analysis and Network Investigation. Front Pharmacol. 2019;10:253.

84. Zhang M-Y, Xu L-P, Yang H-J. Fructus and its active ingredients as promising resources for the treatment of neurological diseases. Int J Mol Sci. 2018;19(7):1970.

85. Chen $\mathrm{X}, \mathrm{CaO} \mathrm{J}-\mathrm{H}$, Sun $\mathrm{Y}$, et al. Ethanol extract of Schisandrae chinensis fructus ameliorates the extent of experimentally induced atherosclerosis in rats by increasing antioxidant capacity and improving endothelial dysfunction. Pharm Biol. 2018;56(1):612-9.

86. Yang JY, Kim GR, Chae JS, et al. Antioxidant and anti-inflammatory effects of an ethanol fraction from the baillon hot water extract fermented using subsp. Food Sci Biotechnol. 2019;28(6):1759-67.

87. Karna KK, Choi BR, Kim M-J, et al. The Effect of Schisandra chinensis Baillon on Cross-Talk between Oxidative Stress, Endoplasmic Reticulum Stress, and Mitochondrial Signaling Pathway in Testes of Varicocele-Induced SD Rat. Int J Mol Sci. 2019;20(22):5785

88. Nowak A, Zakłos-Szyda M, Błasiak J, et al. Potential of (Turcz.) Baill. in Human Health and Nutrition: A Review of Current Knowledge and Therapeutic Perspectives. Nutrients. 2019;11(2):333.

89. Kim HS, Lee JH, Park HS, et al. Schizandra chinensis extracts induce apoptosis in human gastric cancer cells via JNK/p38 MAPK activation and the ROS-mediated/mitochondria-dependent pathway. Pharm Biol. 2015; 53(2):212-9.

90. Lai Q, Yuan G-Y, Wang $H$, et al. Exploring the protective effects of schizandrol a in acute myocardial ischemia mice by comprehensive metabolomics profiling integrated with molecular mechanism studies. Acta Pharmacol Sin. 2020:41(8):1058-72.

91. Li H-Y, Fang J-J, Shen H-D, et al. "Quantity-effect" research strategy for comparison of antioxidant activity and quality of Rehmanniae Radix and Rehmannia Radix Praeparata by on-line HPLC-UV-ABTS assay. BMC Complementary Medicine and Therapies. 2020;20(1):16.

92. Zhang X, Wang D-D, Ren X-D, et al. System Bioinformatic approach through molecular docking, network pharmacology and microarray data analysis to determine the molecular mechanism underlying the effects of Rehmanniae Radix Praeparata on cardiovascular diseases. Curr Protein Pept Sci. 2019; 20(10):964-75.

93. Wan $\mathrm{G}$, Zhang N-D, Cheng G, et al. Libosch extracts prevent bone loss and architectural deterioration and enhance Osteoblastic bone formation by regulating the IGF-1/PI3K/mTOR pathway in Streptozotocin-induced diabetic rats. Int J Mol Sci. 2019;20(16):3964.

94. Meim X-D, Cao Y-F, Che Y-Y, et al. Danshen: a phytochemical and pharmacological overview. Chin J Nat Med. 2019:17(1):59-80.

95. Kim H-G, Lee J-S, Choi M-K, et al. Ethanolic extract of Astragali radix and Salviae radix prohibits oxidative brain injury by psycho-emotional stress in whisker removal rat model. PLoS One. 2014;9(5):e98329.

96. Zhang X-P, Li Z-J, Liu D-R. Progress in research into the mechanism of Radix salviae miltiorrhizae in treatment of acute pancreatitis. Hepatobiliary \& Pancreatic Diseases Int. 2006:5(4):501-4.

97. Li G-H, Li Y-R, Jiao P, et al. Therapeutic Potential of Salviae Miltiorrhizae Radix et Rhizoma against Human Diseases Based on Activation of Nrf2Mediated Antioxidant Defense System: Bioactive Constituents and Mechanism of Action. Oxidative Medicine and Cellular Longevity. 2018;2018: 7309073.

98. Lu J, Song H-P, Li P, et al. Screening of direct thrombin inhibitors from Radix Salviae Miltiorrhizae by a peak fractionation approach. J Pharm Biomed Anal. 2015;109:85-90

99. Zhang $X-W$, Lin $Y$, Lin $A$, et al. Discovery of cancer cell proliferation inhibitors from Salviae miltiorrhizae radix et rhizoma by a trace peak enrichment approach. J Sep Sci. 2019:42(2):534-46.

100. Tzeng H-E, Tsai C-H, Ho T-Y, et al. Radix Paeoniae Rubra stimulates osteoclast differentiation by activation of the NF-KB and mitogen-activated protein kinase pathways. BMC Complement Altern Med. 2018;18(1):132.

101. Lin M-Y, Chiang S-Y, Li Y-Z, et al. Anti-tumor effect of Radix Paeoniae Rubra extract on mice bladder tumors using intravesical therapy. Oncol Lett. 2016; 12(2):904-10.
102. Ke Z-C, Wang G, Yang L, et al. Crude terpene glycoside component from Radix paeoniae rubra protects against isoproterenol-induced myocardial ischemic injury via activation of the PI3K/AKT/mTOR signaling pathway. J Ethnopharmacol. 2017;206:160-9.

103. Huang $W$-J, Lin L, Tian X-P, et al. Astragalus and Paeoniae radix rubra extract inhibits liver fibrosis by modulating the transforming growth factor- $\beta / S$ mad pathway in rats. Mol Med Rep. 2015;11(2):805-14.

104. Zhong L-J, Xie Z-S, Yang H, et al. Moutan cortex and Paeoniae Radix Rubra reverse high-fat-diet-induced metabolic disorder and restore gut microbiota homeostasis. Chin J Nat Med. 2017;15(3):210-9.

105. Xie P-Y, Cui L-L, Shan Y, et al. Antithrombotic Effect and Mechanism of Radix Paeoniae Rubra. BioMed Research Int. 2017;2017:9475074.

106. Lan L-L, Zhang Y-J, Zhang M-T, et al. Evaluation of the quality of compound liquorice tablets by DSC and HPLC fingerprints assisted with dissolution. J Pharmaceutical Biomedical Analysis. 2019:175:112715.

107. Liang C-H, Wang X-C, Hu J-P, et al. PTPRO promotes oxidized low-density lipoprotein induced oxidative stress and cell apoptosis through toll-like receptor 4/nuclear factor KB pathway. Cell Physiol Biochem. 2017;42(2):495505.

108. Chen R, Ning G, Gao Y, et al. TLR4 Asp299Gly (rs4986790) polymorphism and coronary artery disease: a meta-analysis. PeerJ. 2015;3:e1412.

\section{Publisher's Note}

Springer Nature remains neutral with regard to jurisdictional claims in published maps and institutional affiliations.

\section{Ready to submit your research? Choose BMC and benefit from:}

- fast, convenient online submission

- thorough peer review by experienced researchers in your field

- rapid publication on acceptance

- support for research data, including large and complex data types

- gold Open Access which fosters wider collaboration and increased citations

- maximum visibility for your research: over $100 \mathrm{M}$ website views per year

At BMC, research is always in progress.

Learn more biomedcentral.com/submissions 